\title{
APOSYNDETIC PROPERTIES OF THE $n$-FOLD SYMMETRIC PRODUCT SUSPENSION OF A CONTINUUM
}

\author{
FRANCO BARRAGÁN
}

Universidad Tecnológica de la Mixteca, Mexico

\begin{abstract}
In this paper the $n$-fold symmetric product suspension of a continuum is investigated with respect to the properties of aposyndesis such as: aposyndesis, finite aposyndesis, mutual aposyndesis and strictly nonmutual aposyndesis.
\end{abstract}

\section{INTRODUCTION}

In $1941 \mathrm{~F}$. B. Jones introduced the notion of aposyndesis ([8]). In [6, Theorem 1], [11, Theorem 4], [12, Corollary 5.2], [13, Corollary 4.3], [15, Corollary 2.36] and [7, Theorem 2.4] we can find some results of aposyndesis in hyperspaces of continua. Given a continuum $X$ and an integer $n \geq 1$, in 1931 K. Borsuk and S. Ulam ([4]) defined the $n$-fold symmetric product of $X, F_{n}(X)=\{A \subset X: A$ has at most $n$ points $\}$. For each integer $n \geq 2$, in the year 2009 we defined the $n$-fold symmetric product suspension of $X$ ([1]), denoted by $S F_{n}(X)$, as the quotient space $F_{n}(X) / F_{1}(X)$. In [2] we investigated the induced maps between these spaces.

In $1997 \mathrm{~S}$. Macías showed in [11, Theorem 4], that $F_{n}(X)$ is colocally connected (in particular aposyndetic), for every $n \geq 2$. In [11, Theorem 8] he also proved that $F_{n}(X)$ is countable closed aposyndetic, for every $n \geq 2$. In the present paper, we prove that $S F_{n}(X)$ is colocally connected (in particular aposyndetic), for every $n \geq 3$. Also we prove that $F_{n}(X)$ is finitely aposyndetic, for every $n \geq 3$. Furthermore, we show a class of continua for which their second symmetric product suspensions are not

2010 Mathematics Subject Classification. 54B20, 54F15.

Key words and phrases. Aposyndetic continuum, chainable continuum, mutually aposyndetic, symmetric product, strictly nonmutually aposyndetic. 
aposyndetic. Moreover, we prove that if a continuum is aposyndetic, then its second symmetric product suspension is aposyndetic.

On the other hand, in 1999 J. M. Martinez-Motejano showed in [16, Theorem 1] that $F_{n}(X)$ is mutually aposyndetic, for every $n \geq 3$. In [11, Theorem 15] S. Macías proved that if $X$ is a chainable continuum such that its second symmetric product is mutually aposyndetic, then $X$ is the arc. In this paper we prove that $S F_{n}(X)$ is mutually aposyndetic, for every $n \geq 3$. Also we show that if a continuum is aposyndetic, then its second symmetric product is mutually aposyndetic. Furthermore, we proved that if a continuum is 2-aposyndetic and mutually aposyndetic, then its second symmetric product suspension is mutually aposyndetic. Moreover, we verify that if $X$ is a chainable continuum such that its second symmetric product suspension is mutually aposyndetic, then $X$ is the arc.

In [11, Theorem 16] S. Macías proved that a chainable continuum is indecomposable if and only if its second symmetric product is strictly nonmutually aposyndetic. We show that a continuum with span zero is indecomposable if and only if its second symmetric product suspension is strictly nonmutually aposyndetic.

\section{Definitions}

The symbol $\mathbb{N}$ will denote the set of positive integers. A continuum is a nonempty compact, connected metric space. A subcontinuum is a continuum contained in a space $X$. If $X$ is a continuum, then given $A \subset X$ and $\epsilon>0$, the open ball around $A$ of radius $\epsilon$ is denoted by $N(\epsilon, A)$, the closure of $A$ in $X$ by $C l_{X}(A)$, the interior of $A$ in $X$ is denoted by $i n t_{X}(A)$ and the boundary of $A$ in $X$ is denoted by $B d(A)$. If $A=\{a\}$, we let $N(a, \epsilon)$ denote the open ball around $a$ of radius $\epsilon$. An arc is any space which is homeomorphic to the closed interval $[0,1]$. A ray is a space homeomorphic to $[0, \infty)$. An Elsa continuum, denoted E-continuum, is a compactification of the ray with an arc as the remainder. A map means a continuous function. An onto map $f: X \rightarrow Y$ between continua is said to be:

- monotone provided that $f^{-1}(y)$ is connected for each $y \in Y$;

- open provided that if $U$ is any open subset of $X$, then $f(U)$ is an open subset of $Y$.

A continuum $X$ is said to be colocally connected, provided that each point of it has a local base of open sets whose complements are connected. A continuum $X$ is said to be aposyndetic at $x$ with respect to $y$, provided that there exists a subcontinuum $W$ of $X$ such that $x \in$ int $_{X}(W) \subset W \subset X \backslash\{y\}$, it is said to be aposyndetic at $x$, if it is aposyndetic at $x$ with respect to any point of $X \backslash\{x\}$, and it is said to be aposyndetic, if it is aposyndetic at each of its points. A continuum $X$ is 2-aposyndetic at $x$ if for every pair of points $p$ and $q$ lying in $X \backslash\{x\}$, there exists a subcontinuum $W$ of $X$ such 
that $x \in \operatorname{int}_{X}(W) \subset W \subset X \backslash\{p, q\}$. A continuum is 2-aposyndetic if it is 2-aposyndetic at every one of its points. Let $\mathcal{F}$ be a collection of finite subsets of a continuum $X$. Then $X$ is said to be finitely aposyndetic if for each $x \in X$ and each $F \in \mathcal{F}$ such that $x \notin F$, there exists a subcontinuum $W$ of $X$ such that $x \in \operatorname{int}_{X}(W)$ and $W \cap F=\emptyset$. A continuum $X$ is mutually aposyndetic, provided that if $x$ and $y$ are two distinct points of $X$ then there exist two disjoint subcontinua $W_{x}$ and $W_{y}$ of $X$ such that $x \in \operatorname{int}_{X}\left(W_{x}\right)$ and $y \in \operatorname{int}_{X}\left(W_{y}\right)$. A continuum $X$ is said to be strictly nonmutually aposyndetic if each pair of subcontinua of $X$ which have nonempty interior intersect. A point $x$ of a continuum $X$ is called a cut point of $X$, provided that $X \backslash\{x\}$ is not connected. A point $x$ of a continuum $X$ is called a not-cut point of $X$, provided that $X \backslash\{x\}$ is connected.

A chain in a continuum $X$ is a finite collection $\left\{U_{1}, U_{2}, \ldots, U_{m}\right\}$ of open subsets of $X$ such that $U_{i} \cap U_{j} \neq \emptyset$ if and only if $|i-j| \leq 1$ for each $i, j \in$ $\{1,2, \ldots, m\}$. The elements of a chain are called links. For $\epsilon>0$ an $\epsilon$-chain is a chain in which each link has diameter less than $\epsilon$. A continuum is chainable if for each $\epsilon>0$, it can be covered by an $\epsilon$-chain. A continuum is decomposable provided that it can be written as the union of two of its proper subcontinua. A continuum is indecomposable if it is not decomposable.

Given a continuum $X$ and $n \in \mathbb{N}$, the product of $X$ with itself $n$ times will be denoted by $X^{n}$. The symbol $\Delta_{X^{2}}$ will denote the diagonal of $X^{2}$, that is, $\Delta_{X^{2}}=\left\{(x, x) \in X^{2}: x \in X\right\}$. If $W$ is a subset of $X^{2}$, we let $W^{*}$ denote the subset $\left\{(y, x) \in X^{2}:(x, y) \in W\right\}$ of $X^{2}$. Denote the first and second projections of $X^{2}$ onto $X$ by $\pi_{1}$ and $\pi_{2}$, respectively. The span of a continuum $(X, d)$ (respectively, the semispan of $X$ ), denote by $\sigma(X)$ (respectively, $\sigma_{0}(X)$ ), is the least upper bound of the set of all numbers $\epsilon \geq 0$ for which there exists a subcontinuum $Z$ of $X^{2}$ such that $\pi_{1}(Z)=\pi_{2}(Z)$ (respectively, $\pi_{2}(Z) \subset \pi_{1}(Z)$ ) and $d(x, y) \geq \epsilon$ for each $(x, y) \in Z$.

Given a continuum $X$ and $n \in \mathbb{N}$, the $n$-fold symmetric product of $X$, denoted by $F_{n}(X)$, is the hyperspace:

$$
F_{n}(X)=\{A \subset X: A \text { has at most } n \text { points }\}
$$

topologized with the Hausdorff metric, which is defined as follows:

$$
\mathcal{H}(A, B)=\inf \{\epsilon>0: A \subset N(\epsilon, B) \text { and } B \subset N(\epsilon, A)\} .
$$

On the other hand, $F_{n}(X)$ can be topologized with the topology defined as follows: given a finite collection, $U_{1}, \ldots, U_{m}$, of open sets of $X,\left\langle U_{1}, \ldots, U_{m}\right\rangle_{n}$, denotes the following subset of $F_{n}(X)$ :

$$
\left\{A \in F_{n}(X): A \subset \bigcup_{k=1}^{m} U_{k} \text { and } A \cap U_{k} \neq \emptyset \text { for each } k \in\{1, \ldots, m\}\right\} .
$$

It is known that the family of all subset of $F_{n}(X)$ of the form $\left\langle U_{1}, \ldots, U_{m}\right\rangle_{n}$, as defined above, form a basis for a topology for $F_{n}(X)$ (see $[18,0.11]$ ) called 
the Vietoris topology, and that the Vietoris topology and the topology induced by the Hausdorff metric coincide [18, 0.13].

Given a continuum $X$ and $n \in \mathbb{N}$ with $n \geq 2$, we defined (in [1]) the $n$-fold symmetric product suspension of the continuum $X$, denoted by $S F_{n}(X)$, as the quotient space:

$$
S F_{n}(X)=F_{n}(X) / F_{1}(X),
$$

with the quotient topology.

Give a continuum $X$ and an integer $n \geq 2, q_{X}^{n}: F_{n}(X) \rightarrow S F_{n}(X)$ denotes the quotient map. Also, let $F_{X}^{n}$ denotes the point $q_{X}^{n}\left(F_{1}(X)\right)$. We denote by $f_{2}: X^{2} \rightarrow F_{2}(X)$ the map given by $f_{2}(x, y)=\{x, y\}$, for each $(x, y) \in X^{2}$.

REMARK 2.1. Let $X$ be a continuum and let $n \geq 2$ be an integer. Then $S F_{n}(X) \backslash\left\{F_{X}^{n}\right\}$ is homeomorphic to $F_{n}(X) \backslash F_{1}(X)$.

\section{Aposyndesis}

Theorem 3.1. Let $X$ be a continuum and let $n \geq 2$ be an integer. Then (1) and (2) below are true.

(1) $S F_{n}(X)$ is aposyndetic at $F_{X}^{n}$.

(2) If $\mathcal{A} \in S F_{n}(X) \backslash\left\{F_{X}^{n}\right\}$, then $S F_{n}(X)$ is aposyndetic at $\mathcal{A}$ respect to any point of $S F_{n}(X) \backslash\left\{F_{X}^{n}, \mathcal{A}\right\}$.

Proof. To prove (1), let $\mathcal{B} \in S F_{n}(X) \backslash\left\{F_{X}^{n}\right\}$. Let $\Gamma$ and $\Lambda$ be open subsets of $S F_{n}(X)$ such that $F_{X}^{n} \in \Gamma, \mathcal{B} \in \Lambda$ and $\Gamma \cap \Lambda=\emptyset$. Let $B \in$ $F_{n}(X) \backslash F_{1}(X)$ be such that $q_{X}^{n}(B)=\mathcal{B}$. Hence, $\left(q_{X}^{n}\right)^{-1}(\Lambda)$ is an open subset of $F_{n}(X)$ such that $B \in\left(q_{X}^{n}\right)^{-1}(\Lambda) \subset F_{n}(X) \backslash F_{1}(X)$. By [11, Theorem 4], there exists an open subset $\mathcal{U}$ of $F_{n}(X)$ such that $B \in \mathcal{U} \subset\left(q_{X}^{n}\right)^{-1}(\Lambda)$ and $F_{n}(X) \backslash \mathcal{U}$ is connected. It follows that $\mathcal{B} \in q_{X}^{n}(\mathcal{U}) \subset \Lambda$ and $q_{X}^{n}\left(F_{n}(X) \backslash \mathcal{U}\right)$ is connected. Since $\mathcal{U} \cap F_{1}(X)=\emptyset$, by Remark 2.1 , we have that $q_{X}^{n}(\mathcal{U})$ is an open subset of $S F_{n}(X)$ and $q_{X}^{n}\left(F_{n}(X) \backslash \mathcal{U}\right)=S F_{n}(X) \backslash q_{X}^{n}(\mathcal{U})$. Then $S F_{n}(X) \backslash q_{X}^{n}(\mathcal{U})$ is a subcontinuum of $S F_{n}(X)$. We note that $F_{X}^{n} \in \Gamma \subset S F_{n}(X) \backslash q_{X}^{n}(\mathcal{U})$, thus $F_{X}^{n} \in \operatorname{int}_{S F_{n}(X)}\left(S F_{n}(X) \backslash q_{X}^{n}(\mathcal{U})\right)$. Therefore, $S F_{n}(X) \backslash q_{X}^{n}(\mathcal{U})$ is a subcontinuum of $S F_{n}(X)$ such that $F_{X}^{n} \in \operatorname{int}_{S F_{n}(X)}\left(S F_{n}(X) \backslash q_{X}^{n}(\mathcal{U})\right) \subset$ $S F_{n}(X) \backslash q_{X}^{n}(\mathcal{U}) \subset S F_{n}(X) \backslash\{\mathcal{B}\}$. Hence, $S F_{n}(X)$ is aposyndetic at $F_{X}^{n}$.

A similar argument can be used to verify (2).

Theorem 3.2. Let $X$ be a continuum and let $n \geq 3$ be an integer. Then $S F_{n}(X)$ is aposyndetic.

Proof. By Theorem 3.1, it is sufficient to prove that, for each element $\mathcal{A} \in S F_{n}(X) \backslash\left\{F_{X}^{n}\right\}, S F_{n}(X)$ is aposyndetic at $\mathcal{A}$ with respect to $F_{X}^{n}$. Let $\mathcal{A} \in S F_{n}(X) \backslash\left\{F_{X}^{n}\right\}$. We take $A \in F_{n}(X) \backslash F_{1}(X)$ such that $q_{X}^{n}(A)=$ $\mathcal{A}$. Fix points $p, q \in A$ with $p \neq q$. Let $U$ and $V$ be open subsets of $X$ such that $p \in U, q \in V, C l_{X}(U) \cap C l_{X}(V)=\emptyset$ and $A \subset U \cup V$. We define $\mathcal{C}=\left\langle C l_{X}(U), C l_{X}(V), X\right\rangle_{n} \cup\langle B d(U), B d(V), X\rangle_{n} \cup\langle B d(U),\{p\}, X\rangle_{n} \cup$ 
$\langle\{p\},\{q\}, X\rangle_{n}$. By [16, Lemma 2], $\mathcal{C}$ is a subcontinuum of $F_{n}(X)$. Since $A \in\langle U, V\rangle_{n} \subset \mathcal{C}$, we obtain that $A \in \operatorname{int}_{F_{n}(X)}(\mathcal{C}) \subset \mathcal{C}$. Moreover, we note that $\mathcal{C} \cap F_{1}(X)=\emptyset$. Hence, by Remark $2.1, q_{X}^{n}(\mathcal{C})$ is a subcotinuum of $S F_{n}(X)$ such that $\mathcal{A} \in \operatorname{int}_{S F_{n}(X)}\left(q_{X}^{n}(\mathcal{C})\right) \subset q_{X}^{n}(\mathcal{C}) \subset S F_{n}(X) \backslash\left\{F_{X}^{n}\right\}$. Consequently, $S F_{n}(X)$ is aposyndetic at $\mathcal{A}$ with respect to $F_{X}^{n}$.

A continuum $X$ is said to be unicoherent provided that whenever $A$ and $B$ are proper subcontinua of $X$ with $X=A \cup B$, then $A \cap B$ is connected. We proved in [1, Theorem 4.1] that for each integer $n \geq 3, S F_{n}(X)$ is unicoherent. Moreover, it is known that a unicoherent aposyndetic continuum is finitely aposyndetic ([3]). Therefore, by Theorem 3.2 and [1, Theorem 4.1], we have the following corollary.

Corollary 3.3. Let $X$ be a continuum and let $n \geq 3$ be an integer. Then $S F_{n}(X)$ is finitely aposyndetic.

LEMMA 3.4. Let $X$ be a continuum and let $n \geq 2$ be an integer. Then $F_{n}(X) \backslash F_{1}(X)$ is connected.

Proof. We consider two cases.

(1) First we consider $n=2$. Respect to $X$, we have two possibilities.

(1.1) The continuum $X$ is the arc. Then $F_{2}(X) \backslash F_{1}(X)$ is connected.

(1.2) The continuum $X$ is not the arc. Then, by [9, Theorem 2], it follows that $F_{2}(X) \backslash F_{1}(X)$ is connected.

(2) Now we consider $n \geq 3$. Let $A, B \in F_{n}(X) \backslash F_{1}(X)$ be such that $A \neq B$. Without loss of generality, suppose that there exists a point $b \in B \backslash A$. Take $c \in B \backslash\{b\}$. Fix points $x, y \in A$ with $x \neq y$. Let $U$ and $V$ be open subsets of $X$ such that $x \in U, y \in V, b \notin C l_{X}(U), C l_{X}(U) \cap C l_{X}(V)=\emptyset$ and $A \subset U \cup V$. Let $\mathcal{C}=\left\langle C l_{X}(U), C l_{X}(V), X\right\rangle_{n} \cup\langle B d(U), B d(V), X\rangle_{n} \cup\langle B d(U),\{b\}, X\rangle_{n} \cup$ $\langle\{b\},\{c\}, X\rangle_{n}$. By [16, Lemma 2], $\mathcal{C}$ is connected. Furthermore, $\mathcal{C} \subset F_{n}(X) \backslash$ $F_{1}(X), A \in\left\langle C l_{X}(U), C l_{X}(V), X\right\rangle_{n}$ and $B \in\langle\{b\},\{c\}, X\rangle_{n}$. Therefore, $\mathcal{C}$ is a connected subset of $F_{n}(X) \backslash F_{1}(X)$ such that $A, B \in \mathcal{C}$.

Theorem 3.5. Let $X$ be a continuum and let $n \geq 2$ be an integer. Then each point of $S F_{n}(X)$ is a not-cut point of $S F_{n}(X)$.

Proof. Let $\mathcal{A} \in S F_{n}(X)$. We have the following cases.

(1) Suppose that $\mathcal{A}=F_{X}^{n}$. By Lemma 3.4, we have that $F_{n}(X) \backslash F_{1}(X)$ is a connected subset of $F_{n}(X)$. Then, by Remark 2.1, $q_{X}^{n}\left(F_{n}(X) \backslash F_{1}(X)\right)=$ $S F_{n}(X) \backslash\left\{F_{X}^{n}\right\}$ is a connected subset of $S F_{n}(X)$. This implies that $\mathcal{A}$ is a not-cut point of $S F_{n}(X)$.

(2) Suppose that $\mathcal{A} \in S F_{n}(X) \backslash\left\{F_{X}^{n}\right\}$. We take $A \in F_{n}(X) \backslash F_{1}(X)$ such that $q_{X}^{n}(A)=\mathcal{A}$. By $\left[11\right.$, Corollary 5], it follows that $F_{n}(X) \backslash\{A\}$ is a connected subset of $F_{n}(X)$. Hence, $q_{X}^{n}\left(F_{n}(X) \backslash\{A\}\right)=S F_{n}(X) \backslash\{\mathcal{A}\}$ is a connected subset of $S F_{n}(X)$. Consequently, we conclude that $\mathcal{A}$ is a not-cut point of $S F_{n}(X)$. 
It is known that if each point of $X$ is a not-cut point of $X$, then the continuum $X$ is colocally connected if and only if $X$ is aposyndetic ([21, 4.14, p. 50]). Hence, by Theorems 3.2 and 3.5, we have the following:

Corollary 3.6. Let $X$ be a continuum and let $n \geq 3$ be an integer. Then $S F_{n}(X)$ is colocally connected.

Lemma 3.7. Let $X$ be a continuum. Then the following are equivalent:

(1) for each point $\left(x_{1}, x_{2}\right) \in X^{2} \backslash \triangle_{X^{2}}$, there exists a subcontinuum $C$ of $X^{2}$ such that $\left(x_{1}, x_{2}\right) \in$ int $_{X^{2}}(C)$ and $C \cap \triangle_{X^{2}}=\emptyset$,

(2) for each element $\mathcal{A} \in S F_{2}(X) \backslash\left\{F_{X}^{2}\right\}, S F_{2}(X)$ is aposyndetic at $\mathcal{A}$ with respect to $F_{X}^{2}$.

Proof. We prove that (1) implies (2). Let $\mathcal{A} \in S F_{2}(X) \backslash\left\{F_{X}^{2}\right\}$. We take $A \in F_{2}(X) \backslash F_{1}(X)$ such that $q_{X}^{2}(A)=\mathcal{A}$. Suppose that $A=\left\{a_{1}, a_{2}\right\}$. Since $\left(a_{1}, a_{2}\right) \in X^{2} \backslash \triangle_{X^{2}}$, by (1), there exists a subcontinuum $C$ of $X^{2}$ such that $\left(a_{1}, a_{2}\right) \in \operatorname{int}_{X^{2}}(C)$ and $C \cap \triangle_{X^{2}}=\emptyset$. Thus, since the map $f_{2}: X^{2} \rightarrow F_{2}(X)$ given by $f_{2}\left(\left(x_{1}, x_{2}\right)\right)=\left\{x_{1}, x_{2}\right\}$ is an open map ([11, Lemma 9$\left.]\right)$, we obtain that $f_{2}\left(\left(a_{1}, a_{2}\right)\right)=A \in \operatorname{int}_{F_{2}(X)}\left(f_{2}(C)\right)$. Furthermore, since $C \cap \triangle_{X^{2}}=\emptyset$, it follows that $f_{2}(C) \cap F_{1}(X)=\emptyset$. Hence, by Remark 2.1, $q_{X}^{2}\left(f_{2}(C)\right)$ is a subcontinuum of $S F_{2}(X)$ such that $\mathcal{A} \in \operatorname{int}_{S F_{2}(X)}\left(q_{X}^{2}\left(f_{2}(C)\right)\right) \subset q_{X}^{2}\left(f_{2}(C)\right) \subset$ $S F_{2}(X) \backslash\left\{F_{X}^{2}\right\}$. This proves that $S F_{2}(X)$ is aposyndetic at $\mathcal{A}$ with respect to $F_{X}^{2}$.

Next we prove that (2) implies (1). Let $\left(a_{1}, a_{2}\right) \in X^{2} \backslash \triangle_{X^{2}}$. We define $A=\left\{a_{1}, a_{2}\right\}$. Then $f_{2}\left(\left(a_{1}, a_{2}\right)\right)=A \in F_{2}(X) \backslash F_{1}(X)$. Let $\mathcal{A}=q_{X}^{2}(A)$. Thus, $\mathcal{A} \in S F_{2}(X) \backslash\left\{F_{X}^{2}\right\}$. By $(2)$, there exists a subcontinuum $\Theta$ of $S F_{2}(X)$ such that $\mathcal{A} \in \operatorname{int}_{S F_{2}(X)}(\Theta) \subset \Theta \subset S F_{2}(X) \backslash\left\{F_{X}^{2}\right\}$. Define $\mathcal{C}=\left(q_{X}^{2}\right)^{-1}(\Theta)$. Since $q_{X}^{2}$ is a monotone map, and by $[21,2.2$, p. 138], $\mathcal{C}$ is a subcontinuum of $F_{2}(X)$, also $A \in \operatorname{int}_{F_{2}(X)}(\mathcal{C})$. Since $F_{X}^{2} \notin \Theta$, we obtain that $\mathcal{C} \cap F_{1}(X)=\emptyset$. This implies that $f_{2}^{-1}(\mathcal{C}) \cap \triangle_{X^{2}}=\emptyset$.

On the other hand, by $[11$, Lemma 12$], f_{2}^{-1}(\mathcal{C})$ has at most two components. Let $f_{2}^{-1}(\mathcal{C})=C_{1} \cup C_{2}$, where $C_{1}$ and $C_{2}$ are the components of $f_{2}^{-1}(\mathcal{C})$. Hence, $C_{1} \cap \triangle_{X^{2}}=\emptyset$ and $C_{2} \cap \triangle_{X^{2}}=\emptyset$. Since $\left(a_{1}, a_{2}\right) \in f_{2}^{-1}(\mathcal{C})$, we suppose without loss of generality, that $\left(a_{1}, a_{2}\right) \in C_{1}$. Since $A \in \operatorname{int}_{F_{2}(X)}(\mathcal{C})$, we obtain that $\left(a_{1}, a_{2}\right) \in \operatorname{int}_{X^{2}}\left(f_{2}^{-1}(\mathcal{C})\right)$. Consequently, by [14, Lemma 1.6.2], we have that $\left(a_{1}, a_{2}\right) \in \operatorname{int}_{X^{2}}\left(C_{1}\right)$. Therefore, $C_{1}$ is a subcontinuum of $X^{2}$ such that $\left(a_{1}, a_{2}\right) \in \operatorname{int}_{X^{2}}\left(C_{1}\right)$ and $C_{1} \cap \triangle_{X^{2}}=\emptyset$.

TheOrem 3.8. If $X$ is an E-continuum, then $S F_{2}(X)$ is not aposyndetic.

Proof. Let $X=J \cup S$, where $J$ is the remainder and $S$ is homeomorphic to $[0, \infty)$. Let $\left(a_{1}, a_{2}\right) \in J \times J$ be such that $\left(a_{1}, a_{2}\right) \in X^{2} \backslash \triangle_{X^{2}}$. Suppose that there exists a subcontinuum $C$ of $X^{2}$ such that $\left(a_{1}, a_{2}\right) \in$ int $_{X^{2}}(C)$. Since the map $\pi_{1}: X^{2} \rightarrow X$, given by $\pi_{1}\left(x_{1}, x_{2}\right)=x_{1}$, is an open map, it follows that $a_{1} \in \operatorname{int}_{X}\left(\pi_{1}(C)\right)$. Hence, since $\pi_{1}(C)$ is a subcontinuum of $X$, we have that 
$J \subset \pi_{1}(C)$. With similar arguments, we obtain that $J \subset \pi_{2}(C)$. Therefore, we have that either $\pi_{1}(C) \subset \pi_{2}(C)$ or $\pi_{2}(C) \subset \pi_{1}(C)$.

On the other hand, by $[17$, Lemma 6, p. 126], $X$ is a chainable continuum. Thus, by [10, p. 210], it follows that $X$ has zero span, and by [5, Theorem 6$]$, $X$ has zero semispan. This implies that $C \cap \triangle_{X^{2}} \neq \emptyset$. Then, by Lemma 3.7, there exists $\mathcal{A} \in S F_{2}(X) \backslash\left\{F_{X}^{2}\right\}$ such that $S F_{2}(X)$ is not aposyndetic at $\mathcal{A}$ with respect to $F_{X}^{2}$. Consequently, $S F_{2}(X)$ is not aposyndetic.

Lemma 3.9. Let $X$ be a continuum, let $x_{1}, x_{2} \in X$ with $x_{1} \neq x_{2}$ and let $A_{1}$ and $A_{2}$ be subcontinua of $X$ such that $x_{1} \in$ int $_{X}\left(A_{1}\right), x_{2} \in$ int $_{X}\left(A_{2}\right), x_{2} \notin A_{1}$ and $x_{1} \notin A_{2}$. Then, for each $\epsilon>0$ with $\epsilon<\frac{1}{2} \min \left\{d\left(x_{1}, A_{2}\right), d\left(x_{2}, A_{1}\right)\right\}$,

$$
C=\left(A_{1} \times A_{2}\right) \backslash\left(N\left(\epsilon, A_{2}\right) \times N\left(\epsilon, A_{1}\right)\right)
$$

is a subcontinuum of $X^{2}$ such that $\left(x_{1}, x_{2}\right) \in$ int $_{X^{2}}(C)$ and $C \cap \triangle_{X^{2}}=\emptyset$.

Proof. Let $\epsilon>0$ be such that $\epsilon<\frac{1}{2} \min \left\{d\left(x_{1}, A_{2}\right), d\left(x_{2}, A_{1}\right)\right\}$. Note that $C$ is a closed subset of $X^{2}$, and thus, $C$ is compact. To prove that $C$ is connected, let $(p, q)$ and $(r, s)$ be two distinct points in $C$, and we will check that there exists a connected subset of $C$ containing the points $(p, q)$ and $(r, s)$. Since $(p, q),(r, s) \in C$, it follows that $p, r \in A_{1}$ and $q, s \in A_{2}$. In order to prove that $C$ is connected, we consider the following cases:

CASE (1). $p \notin N\left(\epsilon, A_{2}\right)$ and $r \notin N\left(\epsilon, A_{2}\right)$. For this case, it follows that $\{p\} \times A_{2}$ and $\{r\} \times A_{2}$ are connected subsets of $C$ such that $(p, q),\left(p, x_{2}\right) \in$ $\{p\} \times A_{2}$ and $(r, s),\left(r, x_{2}\right) \in\{r\} \times A_{2}$. Furthermore, since $x_{2} \notin N\left(\epsilon, A_{1}\right)$, we obtain that $A_{1} \times\left\{x_{2}\right\}$ is a connected subset of $C$ such that $\left(p, x_{2}\right),\left(r, x_{2}\right) \in$ $A_{1} \times\left\{x_{2}\right\}$. We define $K=\left[\{p\} \times A_{2}\right] \cup\left[\{r\} \times A_{2}\right] \cup\left[A_{1} \times\left\{x_{2}\right\}\right]$. Then, $K$ is a connected subset of $C$ containing the points $(p, q)$ and $(r, s)$.

CASE (2). $p \notin N\left(\epsilon, A_{2}\right)$ and $s \notin N\left(\epsilon, A_{1}\right)$. We note that $\{p\} \times A_{2}$ and $A_{1} \times\{s\}$ are connected subsets of $C$ such that $(p, q),(p, s) \in\{p\} \times A_{2}$ and $(p, s),(r, s) \in A_{1} \times\{s\}$. Let $K=\left[\{p\} \times A_{2}\right] \cup\left[A_{1} \times\{s\}\right]$. It follows that $K$ is a connected subset of $C$ such that $(p, q),(r, s) \in K$.

CASE (3). $q \notin N\left(\epsilon, A_{1}\right)$ and $r \notin N\left(\epsilon, A_{2}\right)$. Observe that $A_{1} \times\{q\}$ and $\{r\} \times A_{2}$ are connected subsets of $C$ such that $(p, q),(r, q) \in A_{1} \times\{q\}$ and $(r, s),(r, q) \in\{r\} \times A_{2}$. We define $K=\left[A_{1} \times\{q\}\right] \cup\left[\{r\} \times A_{2}\right]$. Hence, $K$ is a connected subset of $C$ containing the points $(p, q)$ and $(r, s)$.

CASE (4). $q \notin N\left(\epsilon, A_{1}\right)$ and $s \notin N\left(\epsilon, A_{1}\right)$. We obtain that $A_{1} \times\{q\}$ and $A_{1} \times\{s\}$ are connected subsets of $C$ such that $(p, q),\left(x_{1}, q\right) \in A_{1} \times\{q\}$ and $(r, s),\left(x_{1}, s\right) \in A_{1} \times\{s\}$. Since $x_{1} \notin N\left(\epsilon, A_{2}\right)$, we have that $\left\{x_{1}\right\} \times A_{2}$ is a connected subset of $C$ such that $\left(x_{1}, q\right),\left(x_{1}, s\right) \in\left\{x_{1}\right\} \times A_{2}$. We define $K=\left[A_{1} \times\{q\}\right] \cup\left[A_{1} \times\{s\}\right] \cup\left[\left\{x_{1}\right\} \times A_{2}\right]$. It follows that $K$ is a connected subset of $C$ containing the points $(p, q)$ and $(r, s)$.

By the Cases (1)-(4), we conclude that $C$ is connected, and thus, $C$ is a subcontinuum of $X^{2}$. 
On the other hand, we note that $x_{1} \in \operatorname{int}_{X}\left(A_{1}\right) \backslash C l_{X}\left(N\left(\epsilon, A_{2}\right)\right)$ and $x_{2} \in \operatorname{int}_{X}\left(A_{2}\right) \backslash C l_{X}\left(N\left(\epsilon, A_{1}\right)\right)$. Hence, $\left(x_{1}, x_{2}\right) \in i n t_{X^{2}}(C)$. Furthermore, it easy to see that $C \cap \triangle_{X^{2}}=\emptyset$.

THEOREM 3.10. If $X$ is an aposyndetic continuum, then $S F_{2}(X)$ is aposyndetic.

Proof. By Theorem 3.1 and Lemma 3.7, it is sufficient to show that for each point $\left(x_{1}, x_{2}\right) \in X^{2} \backslash \triangle_{X^{2}}$, there exists a subcontinuum $C$ of $X^{2}$ such that $\left(x_{1}, x_{2}\right) \in \operatorname{int}_{X^{2}}(C)$ and $C \cap \triangle_{X^{2}}=\emptyset$. Let $\left(x_{1}, x_{2}\right) \in X^{2} \backslash \triangle_{X^{2}}$. Since $X$ is an aposyndetic continuum, there exist two subcontinua $A_{1}$ and $A_{2}$ of $X$ such that $x_{1} \in \operatorname{int}_{X}\left(A_{1}\right), x_{2} \in \operatorname{int}_{X}\left(A_{2}\right), x_{2} \notin A_{1}$ and $x_{1} \notin A_{2}$. We take $\epsilon>0$ such that $\epsilon<\frac{1}{2} \min \left\{d\left(x_{1}, A_{2}\right), d\left(x_{2}, A_{1}\right)\right\}$. Then, by Lemma 3.9, the subset

$$
C=\left(A_{1} \times A_{2}\right) \backslash\left(N\left(\epsilon, A_{2}\right) \times N\left(\epsilon, A_{1}\right)\right)
$$

is a subcontinuum of $X^{2}$ such that $\left(x_{1}, x_{2}\right) \in i n t_{X^{2}}(C)$ and $C \cap \triangle_{X^{2}}=\emptyset$.

\section{Mutual Aposyndesis}

Lemma 4.1. Let $X$ be a continuum, let $n \geq 2$ be an integer, and let $U$ and $V$ be nonempty, proper, open subsets of $X$ such that $U \subset C l_{X}(U) \subset V$. If $\mathcal{K}=\left\langle C l_{X}(V)\right\rangle_{n} \cup\langle X \backslash U\rangle_{n}$, then $\mathcal{K}$ is a subcontinuum of $F_{n}(X)$ such that $F_{1}(X) \subset \operatorname{int}_{F_{n}(X)}(\mathcal{K})$.

Proof. It is clear that $\mathcal{K}$ is a closed subset of $F_{n}(X)$, and thus $\mathcal{K}$ is compact. Furthermore, since $X=V \cup\left(X \backslash C l_{X}(U)\right)$, it follows that $F_{1}(X) \subset$ $\operatorname{int}_{F_{n}(X)}(\mathcal{K})$.

Next we prove that $\mathcal{K}$ is connected. Since $F_{1}(X)$ is a connected subset of $\mathcal{K}$, it is sufficient to show that for each point $A \in \mathcal{K}$, there exists a connected subset $\mathcal{E}$ of $\mathcal{K}$ such that $A \in \mathcal{E}$ and $\mathcal{E} \cap F_{1}(X) \neq \emptyset$.

Let $A \in \mathcal{K}$. Suppose that $A \in\left\langle C l_{X}(V)\right\rangle_{n}$. We assume that $A=$ $\left\{a_{1}, \ldots, a_{k}\right\}$ with $k \leq n$. For each $i \in\{1, \ldots, k\}$, let $C_{i}$ be the component of $C l_{X}(V)$ such that $a_{i} \in C_{i}$. By [19, Theorem 5.4], for each $i \in\{1, \ldots, k\}$, it follows that $C_{i} \cap B d(V) \neq \emptyset$. Consequently, for each $i \in\{1, \ldots, k\}$, let $b_{i} \in C_{i} \cap B d(V)$. We define $\mathcal{C}=\left\langle C_{1}, \ldots, C_{k}\right\rangle_{n}$ and $B=\left\{b_{1}, \ldots, b_{k}\right\}$. Then, by [16, Lemma 1$], \mathcal{C}$ is a connected set. Moreover, it is clear that $\mathcal{C} \subset \mathcal{K}$ and $A, B \in \mathcal{C}$.

For each $i \in\{1, \ldots, k-1\}$, we define $\mathcal{D}_{i}=\left\{\left\{b_{1}, \ldots, b_{i}\right\} \cup K: K \in F_{1}(X)\right\}$. Note that, for each $i \in\{1, \ldots, k-1\}, \mathcal{D}_{i}$ is a continuum. Furthermore, since for each $i \in\{1, \ldots, k\}, b_{i} \in C l_{X}(V) \cap(X \backslash U)$ and $X=C l_{X}(V) \cup(X \backslash U)$, we obtain that $\mathcal{D}_{1}, \ldots, \mathcal{D}_{k-1}$ are subcontinua of $\mathcal{K}$. Moreover, we have that $\mathcal{D}_{i} \cap$ $\mathcal{D}_{i+1} \neq \emptyset,\left\{b_{1}\right\} \in \mathcal{D}_{1}$ and $B \in \mathcal{D}_{k-1}$. Hence, $\mathcal{D}=\cup_{i=1}^{k-1} \mathcal{D}_{i}$ is a subcontinuum of $\mathcal{K}$ such that $B,\left\{b_{1}\right\} \in \mathcal{D}$. We define $\mathcal{E}=\mathcal{C} \cup \mathcal{D}$. It follows that $\mathcal{E}$ is a subcontinuum of $\mathcal{K}$ such that $A \in \mathcal{E}$ and $\mathcal{E} \cap F_{1}(X) \neq \emptyset$.

Similiarly, we can show the case $A \in\langle X \backslash U\rangle_{n}$. 
Theorem 4.2. Let $X$ be a continuum and let $n \geq 3$ be an integer. Then $S F_{n}(X)$ is mutually aposyndetic.

Proof. Let $\mathcal{A}, \mathcal{B} \in S F_{n}(X)$ be such that $\mathcal{A} \neq \mathcal{B}$. We assume, without loss of generality, that $\mathcal{A} \neq F_{X}^{n}$. We take $A \in F_{n}(X) \backslash F_{1}(X)$ such that $q_{X}^{n}(A)=\mathcal{A}$, and let $B \in F_{n}(X)$ such that $q_{X}^{n}(B)=\mathcal{B}$. Hence, we have the following cases:

CASE (1). $A \not \subset B$. For this case, let $a \in A \backslash B$ and let $b \in A$ with $a \neq b$. We define $B_{1}=B \cup\{b\}$. Let $U, V, U_{1}$ and $V_{1}$ be proper open subsets of $X$ such that $B_{1} \subset U \subset C l_{X}(U) \subset U_{1} \subset C l_{X}\left(U_{1}\right) \subset V_{1} \subset X$, $a \in V$ and $C l_{X}\left(V_{1}\right) \cap C l_{X}(V)=\emptyset$. We define the subsets, $\mathcal{H}$ and $\mathcal{K}$, of $F_{n}(X)$ as follows: $\mathcal{H}=\left\langle C l_{X}(V), C l_{X}(U), X\right\rangle_{n} \cup\langle B d(V), B d(U), X\rangle_{n} \cup$ $\langle B d(V),\{b\}, X\rangle_{n} \cup\langle\{a\},\{b\}, X\rangle_{n}$ and $\mathcal{K}=\left\langle C l_{X}\left(V_{1}\right)\right\rangle_{n} \cup\left\langle X \backslash U_{1}\right\rangle_{n}$. By [16, Lemma 2], $\mathcal{H}$ is a subcontinuum of $F_{n}(X)$. Furthermore, $A \in \operatorname{int}_{F_{n}(X)}(\mathcal{H})$ and $\mathcal{H} \cap F_{1}(X)=\emptyset$. Applying Remark 2.1 , we obtain that $q_{X}^{n}(\mathcal{H})$ is a subcontinuum of $S F_{n}(X)$ such that $\mathcal{A} \in \operatorname{int}_{S F_{n}(X)}\left(q_{X}^{n}(\mathcal{H})\right)$. On the other hand, by Lemma 4.1 , we have that $\mathcal{K}$ is a subcontinuum of $F_{n}(X)$ such that $F_{1}(X) \subset \operatorname{int}_{F_{n}(X)}(\mathcal{K})$. Also, $B \in \operatorname{int}_{F_{n}(X)}(\mathcal{K})$. By Remark 2.1, it follows that $q_{X}^{n}(\mathcal{K})$ is a subcontinuum of $S F_{n}(X)$ such that $\mathcal{B}, F_{X}^{n} \in \operatorname{int}_{S F_{n}(X)}\left(q_{X}^{n}(\mathcal{K})\right)$. Since $\mathcal{H} \cap \mathcal{K}=\emptyset$ and $F_{1}(X) \subset \mathcal{K}$, we obtain that $q_{X}^{n}(\mathcal{H}) \cap q_{X}^{n}(\mathcal{K})=\emptyset$. This proves Case (1).

CAse (2). $A \subset B$. Since $A \in F_{n}(X) \backslash F_{1}(X)$, it follows that $B \in$ $F_{n}(X) \backslash F_{1}(X)$. Since $A \neq B$, we obtain that $B \not \subset A$. Let $b \in B \backslash A$. Since $B \in F_{n}(X) \backslash F_{1}(X)$, there exists a point $a \in B \backslash\{b\}$. Now, we proceed as in Case (1).

Finally, by Cases (1) and (2), we conclude that $S F_{n}(X)$ is mutually aposyndetic.

Theorem 4.3. Let $X$ a chainable continuum. Then $S F_{2}(X)$ is mutually aposyndetic if and only if $X$ is the arc.

Proof. If $X$ is the arc then, by [1, Example 3.1], $S F_{2}(X)$ is homeomorphic to $[0,1]^{2}$, and hence, $S F_{2}(X)$ is mutually aposyndetic .

To prove the converse, by [20, Theorem 1] and [11, Theorem 15], it is sufficient to show that given two different points of the form $(x, q)$ and $(y, q)$ (or $(q, x)$ and $(q, y))$ in $X^{2}$, there exist subcontinua $\mathcal{A}$ and $\mathcal{B}$ of $F_{2}(X)$ such that $f_{2}((x, q)) \in \operatorname{int}_{F_{2}(X)}(\mathcal{A}), f_{2}((y, q)) \in \operatorname{int}_{F_{2}(X)}(\mathcal{B})$ and $\mathcal{A} \cap \mathcal{B}=\emptyset$. To this end, let $(x, q)$ and $(y, q)$ be two different points in $X^{2}$. We denote $A=$ $f_{2}((x, q))$ and $B=f_{2}((y, q))$. Then $q_{X}^{2}(A)$ and $q_{X}^{2}(B)$ are two different points in $S F_{2}(X)$. We take two subcontinua $\Gamma$ and $\Lambda$ of $S F_{2}(X)$ such that $q_{X}^{2}(A) \in$ $\operatorname{int}_{S F_{2}(X)}(\Gamma), q_{X}^{2}(B) \in \operatorname{int}_{S F_{2}(X)}(\Lambda)$ and $\Gamma \cap \Lambda=\emptyset$. Let $\mathcal{A}=\left(q_{X}^{2}\right)^{-1}(\Gamma)$ and let $\mathcal{B}=\left(q_{X}^{2}\right)^{-1}(\Lambda)$. Since $q_{X}^{2}$ is a monotone map, by [21, 2.2, p.138], we obtain that $\mathcal{A}$ and $\mathcal{B}$ are subcontinua of $F_{2}(X)$. Furthermore, $A \in \operatorname{int}_{F_{2}(X)}(\mathcal{A})$, 
$B \in \operatorname{int}_{F_{2}(X)}(\mathcal{B})$ and $\mathcal{A} \cap \mathcal{B}=\emptyset$. Then, proceeding as in [11, Theorem 15] and $[20$, Theorem 1], we can conclude that $X$ is the arc.

As an easy consequence of [11, Lemma 9], we have the following:

Lemma 4.4. Let $X$ be a continuum and let $\left(a_{1}, a_{2}\right)$ and $\left(b_{1}, b_{2}\right)$ be points in $X^{2}$. If $A$ and $B$ are subcontinua of $X^{2}$ such that $\left(a_{1}, a_{2}\right) \in$ int $_{X^{2}}(A)$, $\left(b_{1}, b_{2}\right) \in \operatorname{int}_{X^{2}}(B)$ and $\left(A \cup A^{*}\right) \cap\left(B \cup B^{*}\right)=\emptyset$, then $f_{2}(A)$ and $f_{2}(B)$ are subcontinua of $F_{2}(X)$ such that $\left\{a_{1}, a_{2}\right\} \in$ int $_{F_{2}(X)}\left(f_{2}(A)\right),\left\{b_{1}, b_{2}\right\} \in$ int $_{F_{2}(X)}\left(f_{2}(B)\right)$ and $f_{2}(A) \cap f_{2}(B)=\emptyset$.

THEOREM 4.5. If $X$ is an aposyndetic continuum, then $F_{2}(X)$ is mutaully aposyndetic.

Proof. Let $A, B \in F_{2}(X)$ such that $A \neq B$. Without loss of generality, suppose that there exists a point $b_{1} \in B \backslash A$. Take $a_{1} \in A$. Then we have the following cases:

CASE (1). $A \in F_{1}(X)$. Let $A_{1}$ be a subcontinuum of $X$ such that $a_{1} \in \operatorname{int}_{X}\left(A_{1}\right)$ and $b_{1} \notin A_{1}$. Thus, there exists an open subset $U$ of $X$ such that $b_{1} \in U \subset C l_{X}(U) \subset X \backslash A_{1}$. We define $C=A_{1} \times A_{1}$ and $K=$ $\left(C l_{X}(U) \times X\right) \cup\left(X \times C l_{X}(U)\right)$. It follows that $C$ and $K$ are subcontinua of $X^{2}$ such that $\left(a_{1}, a_{1}\right) \in$ int $_{X^{2}}(C),\left(b_{1}, b_{2}\right) \in$ int $_{X^{2}}(K)$ (with $b_{2} \in X$ such that $\left.B=\left\{b_{1}, b_{2}\right\}\right)$ and $\left(C \cup C^{*}\right) \cap\left(K \cup K^{*}\right)=\emptyset$. Therefore, by Lemma 4.4, $f_{2}(C)$ and $f_{2}(K)$ are two subcontinua of $F_{2}(X)$ such that $A \in \operatorname{int}_{F_{2}(X)}\left(f_{2}(C)\right)$, $B \in \operatorname{int}_{F_{2}(X)}\left(f_{2}(K)\right)$ and $f_{2}(C) \cap f_{2}(C)=\emptyset$.

$\operatorname{CASE}(2)$. $A \in F_{2}(X) \backslash F_{1}(X)$. Let $A=\left\{a_{1}, a_{2}\right\}$. Since $b_{1} \notin A$, there exist subcontinua $A_{1}$ and $A_{2}$ of $X$ such that $a_{1} \in \operatorname{int}_{X}\left(A_{1}\right), a_{2} \in$ int $_{X}\left(A_{2}\right), b_{1} \notin A_{1}$ and $b_{1} \notin A_{2}$. Let $U$ be an open subset of $X$ such that $b_{1} \in U \subset C l_{X}(U) \subset X \backslash\left(A_{1} \cup A_{2}\right)$. We define $C=A_{1} \times A_{2}$ and $K=$ $\left(C l_{X}(U) \times X\right) \cup\left(X \times C l_{X}(U)\right)$. Then $C$ and $K$ are two subcontinua of $X^{2}$ such that $\left(a_{1}, a_{2}\right) \in \operatorname{int}_{X^{2}}(C),\left(b_{1}, b_{2}\right) \in$ int $_{X^{2}}(K)$ (with $b_{2} \in X$ such that $\left.B=\left\{b_{1}, b_{2}\right\}\right)$ and $\left(C \cup C^{*}\right) \cap\left(K \cup K^{*}\right)=\emptyset$. Hence, by Lemma 4.4, we have that $f_{2}(C)$ and $f_{2}(K)$ are subcontinua of $F_{2}(X)$ such that $A \in \operatorname{int}_{F_{2}(X)}\left(f_{2}(C)\right)$, $B \in \operatorname{int}_{F_{2}(X)}\left(f_{2}(K)\right)$ and $f_{2}(C) \cap f_{2}(K)=\emptyset$.

THEOREM 4.6. If a continuum $X$ is 2-aposyndetic and mutually aposyndetic, then $S F_{2}(X)$ is mutually aposyndetic.

Proof. Let $\mathcal{A}, \mathcal{B} \in S F_{2}(X)$ be such that $\mathcal{A} \neq \mathcal{B}$. Without loss of generality, we suppose that $\mathcal{A} \neq F_{X}^{2}$. Hence, there exists $A \in F_{2}(X) \backslash F_{1}(X)$ such that $q_{X}^{2}(A)=\mathcal{A}$. We put $A=\left\{a_{1}, a_{2}\right\}$. We have the following cases:

$\operatorname{CASE}(1) . \mathcal{B} \neq F_{X}^{2}$. Let $B \in F_{2}(X) \backslash F_{1}(X)$ be such that $q_{X}^{2}(B)=\mathcal{B}$. We assume that $B=\left\{b_{1}, b_{2}\right\}$. Suppose that $a_{1} \in A \backslash B$. Then, since $X$ is 2-aposyndetic, there exist two subcontinua $W_{1}$ and $W_{2}$ of $X$ such that $b_{1} \in$ $\operatorname{int}_{X}\left(W_{1}\right), W_{1} \cap\left\{b_{2}, a_{1}\right\}=\emptyset, b_{2} \in \operatorname{int}_{X}\left(W_{2}\right)$ and $W_{2} \cap\left\{b_{1}, a_{1}\right\}=\emptyset$. We fix a 
number $\epsilon>0$ such that $\epsilon<\frac{1}{2} \min \left\{d\left(b_{2}, W_{1}\right), d\left(a_{1}, W_{1}\right), d\left(b_{1}, W_{2}\right), d\left(a_{1}, W_{2}\right)\right\}$. Applying Lemma 3.9, we obtain that

$$
C=\left(W_{1} \times W_{2}\right) \backslash\left(N\left(\epsilon, W_{2}\right) \times N\left(\epsilon, W_{1}\right)\right)
$$

is a subcontinuum of $X^{2}$ such that $\left(b_{1}, b_{2}\right) \in$ int $_{X^{2}}(C)$ and $C \cap \triangle_{X^{2}}=\emptyset$.

We define $U=N\left(a_{1}, \epsilon\right)$. We note that $C l_{X}(U) \cap\left(N\left(\epsilon, W_{1}\right) \cup N\left(\epsilon, W_{2}\right)\right)=$ $\emptyset$. Hence, $C l_{X}(U) \cap\left(W_{1} \cup W_{2}\right)=\emptyset$. We define

$$
Z=\left(C l_{X}(U) \times X\right) \cup\left(X \times C l_{X}(U)\right) .
$$

It follows that $Z$ is a subcontinuum of $X^{2}$ such that $\left(a_{1}, a_{2}\right) \in$ int $_{X^{2}}(Z)$ and $Z=Z^{*}$. Since $\left(a_{1}, a_{2}\right) \in$ int $_{X^{2}}(Z) \cap\left(X^{2} \backslash \triangle_{X^{2}}\right)$, there exists an open subset $V$ of $X^{2}$ with $\left(a_{1}, a_{2}\right) \in V \subset Z$ and $V \subset X^{2} \backslash \triangle_{X^{2}}$. Moreover, it is easy to see that $\left(C \cup C^{*}\right) \cap\left(Z \cup Z^{*}\right)=\emptyset$. By Lemma 4.4, we obtain that $f_{2}(C)$ and $f_{2}(Z)$ are subcontinua of $F_{2}(X)$ such that $A \in \operatorname{int}_{F_{2}(X)}\left(f_{2}(Z)\right), B \in \operatorname{int}_{F_{2}(X)}\left(f_{2}(C)\right)$ and $f_{2}(C) \cap f_{2}(Z)=\emptyset$. Furthermore, by [11, Lemma 9], $f_{2}(V)$ is an open subset of $F_{2}(X)$ with $A \in f_{2}(V) \subset f_{2}(Z)$ and $f_{2}(V) \cap F_{1}(X)=\emptyset$. Applying Remark 2.1, we conclude that $q_{X}^{2}\left(f_{2}(Z)\right)$ and $q_{X}^{2}\left(f_{2}(C)\right)$ are two subcontinua of $S F_{2}(X)$ such that $\mathcal{A} \in \operatorname{int}_{S F_{2}(X)}\left(q_{X}^{2}\left(f_{2}(Z)\right)\right), \mathcal{B} \in \operatorname{int}_{S F_{2}(X)}\left(q_{X}^{n}\left(f_{2}(C)\right)\right)$ and $S F_{2}(X)\left(q_{X}^{2}\left(f_{2}(C)\right) \cap S F_{2}(X)\left(q_{X}^{n}\left(f_{2}(Z)\right)\right)=\emptyset\right.$.

CASE $(2) . \mathcal{B}=F_{X}^{2}$. Since $X$ is mutually aposyndetic, there exist two subcontinua $W_{1}$ and $W_{2}$ of $X$ such that $a_{1} \in \operatorname{int}_{X}\left(W_{1}\right), a_{2} \in \operatorname{int}_{X}\left(W_{2}\right)$ and $W_{1} \cap W_{2}=\emptyset$. Since $X$ is 2-aposyndetic, for each $x \in X \backslash A$, let $W_{x}$ be a subcontinuum of $X$ such that $x \in \operatorname{int}_{X}\left(W_{x}\right)$ and $W_{x} \cap A=\emptyset$. Then $\left\{\operatorname{int}_{X}\left(W_{x}\right): x \in X \backslash A\right\} \cup\left\{\operatorname{int}_{X}\left(W_{1}\right), \operatorname{int}_{X}\left(W_{2}\right)\right\}$ is an open cover of $X$. Hence, there exist $x_{1}, \ldots, x_{m} \in X \backslash A$ such that $X=\cup_{i=1}^{m}$ int $_{X}\left(W_{x_{i}}\right) \cup\left(\right.$ int $_{X}\left(W_{1}\right) \cup$ $\left.\operatorname{int}_{X}\left(W_{2}\right)\right)$.

We define a subset, $W$, of $X^{2}$ as follows:

$$
W=\cup_{i=1}^{m} W_{x_{i}}^{2} \cup\left(W_{1}^{2} \cup W_{2}^{2}\right) .
$$

Then $W$ is a subcontinuum of $X^{2}$ with $\triangle_{X^{2}} \subset i n t_{X^{2}}(W)$.

Let $J=\left\{i \in\{1, \ldots, m\}: W_{x_{i}} \cap W_{1} \neq \emptyset\right\}$ and let $K=\{i \in\{1, \ldots, m\}$ : $\left.W_{x_{i}} \cap W_{2} \neq \emptyset\right\}$. We note $J \neq \emptyset$ and $K \neq \emptyset$.

Let $\epsilon_{1}>0$ and $\epsilon_{2}>0$ be such that $\epsilon_{1}<\frac{1}{2} \min \left\{d\left(W_{x_{i}}, a_{1}\right): i \in\right.$ $\{1, \ldots, m\}\}$ and $\epsilon_{2}<\frac{1}{2} \min \left\{d\left(W_{x_{i}}, a_{2}\right): i \in\{1, \ldots, m\}\right\}$. Let $\epsilon>0$ be such that $\epsilon<\min \left\{\epsilon_{1}, \epsilon_{2}\right\}$. We define a subset, $Z$, of $X$ as $Z=\cup_{i=1}^{m} W_{x_{i}}$. We note that $a_{1} \notin N(\epsilon, Z)$. To see that $a_{1} \notin N(\epsilon, Z)$, suppose that $a_{1} \in N(\epsilon, Z)$. Then there exists $z \in Z$ such that $d\left(a_{1}, z\right)<\epsilon$. Thus, there exists $j \in\{1, \ldots, m\}$ such that $z \in W_{x_{j}}$, so that $\epsilon<d\left(a_{1}, W_{x_{j}}\right) \leq d\left(a_{1}, z\right)<\epsilon$, which is a contradiction. Therefore, $a_{1} \notin N(\epsilon, Z)$. Similarly, $a_{2} \notin N(\epsilon, Z)$.

Let $U_{1}=\operatorname{int}_{X}\left(W_{1}\right) \backslash N(\epsilon, Z)$, and let $U_{2}=i n t_{X}\left(W_{2}\right) \backslash N(\epsilon, Z)$. It follows that $U_{1} \times U_{2}$ is an open subset of $X^{2}$ such that $\left(a_{1}, a_{2}\right) \in U_{1} \times U_{2}$. We define

$$
C=\left(W_{1} \times W_{2}\right) \backslash(N(\epsilon, Z) \times N(\epsilon, Z)) .
$$


Since $U_{1} \times U_{2} \subset C$, we obtain that $\left(a_{1}, a_{2}\right) \in$ int $_{X^{2}}(C)$. Moreover, we have that $C$ is a closed subset of $X$, and thus, $C$ is compact.

Next, we prove that $C$ is connected. Let $(p, q)$ and $(r, s)$ be two distinct points in $C$. We are going to show that there is a connected subset in $C$ containing $(p, q)$ and $(r, s)$. Since $(p, q),(r, s) \in C$, it follows that $(p, q),(r, s) \in$ $W_{1} \times W_{2}$ and $(p, q),(r, s) \notin N(\epsilon, Z) \times N(\epsilon, Z)$. Hence, $p, r \in W_{1}, q, s \in W_{2}$, $[p \notin N(\epsilon, Z)$ or $q \notin N(\epsilon, Z)]$ and $[r \notin N(\epsilon, Z)$ or $s \notin N(\epsilon, Z)]$. Consequently, we have the following possibilities:

(a) $p \notin N(\epsilon, Z)$ and $r \notin N(\epsilon, Z)$. In this case, it follows that $\{p\} \times W_{2}$ and $\{r\} \times W_{2}$ are two connected subsets of $C$ such that $(p, q),\left(p, a_{2}\right) \in\{p\} \times W_{2}$ and $(r, s),\left(r, a_{2}\right) \in\{r\} \times W_{2}$. Moreover, since $a_{2} \notin N(\epsilon, Z)$, we have that $W_{1} \times\left\{a_{2}\right\}$ is a connected subset of $C$ such that $\left(p, a_{2}\right),\left(r, a_{2}\right) \in W_{1} \times\left\{a_{2}\right\}$. We define $D=\left[\{p\} \times W_{2}\right] \cup\left[\{r\} \times W_{2}\right] \cup\left[W_{1} \times\left\{a_{2}\right\}\right]$. Then $D$ is a connected subset of $C$ containing $(p, q)$ and $(r, s)$.

(b) $p \notin N(\epsilon, Z)$ and $s \notin N(\epsilon, Z)$. It follows that $\{p\} \times W_{2}$ and $W_{1} \times\{s\}$ are two connected subsets of $C$ such that $(p, q),(p, s) \in\{p\} \times W_{2}$ and $(p, s),(r, s) \in$ $W_{1} \times\{s\}$. We define $D=\left[\{p\} \times W_{2}\right] \cup\left[W_{1} \times\{s\}\right]$. Then $D$ is a connected subset of $C$ containing $(p, q)$ and $(r, s)$.

(c) $q \notin N\left(\epsilon, A_{1}\right)$ and $r \notin N\left(\epsilon, A_{2}\right)$. This case is similar to (b), with $D=\left[W_{1} \times\{q\}\right] \cup\left[\{r\} \times W_{2}\right]$.

(d) $q \notin N\left(\epsilon, A_{1}\right)$ and $s \notin N\left(\epsilon, A_{1}\right)$.

This case is similar to (a), with $D=\left[W_{1} \times\{q\}\right] \cup\left[W_{1} \times\{s\}\right] \cup\left[\left\{a_{1}\right\} \times W_{2}\right]$.

By (a)-(d), we conclude that $C$ is a connected subset of $X^{2}$. Consequently, $C$ is a subcontinuum of $X^{2}$ such that $\left(a_{1}, a_{2}\right) \in \operatorname{int}_{X^{2}}(C)$.

Next we prove that $C \cap W=\emptyset$. Suppose that $\left(c_{1}, c_{2}\right) \in C \cap W$. This implies that $\left(c_{1}, c_{2}\right) \in W_{1} \times W_{2},\left(c_{1}, c_{2}\right) \notin N(\epsilon, Z) \times N(\epsilon, Z)$ and $\left(c_{1}, c_{2}\right) \in W$. Since $W=\cup_{i=1}^{m} W_{x_{i}}^{2} \cup\left(W_{1}^{2} \cup W_{2}^{2}\right)$, we have that either $\left(c_{1}, c_{2}\right) \in W_{1}^{2}$ or $\left(c_{1}, c_{2}\right) \in W_{2}^{2}$ or there exists $i \in\{1, \ldots, r\}$ such that $\left(c_{1}, c_{2}\right) \in W_{x_{i}}^{2}$. If $\left(c_{1}, c_{2}\right) \in W_{1}^{2}$, then $c_{2} \in W_{1} \cap W_{2}$, which is a contradiction. If $\left(c_{1}, c_{2}\right) \in W_{2}^{2}$, then $c_{1} \in W_{1} \cap W_{2}$, which is a contradiction. If there exists $i \in\{1, \ldots, r\}$ such that $\left(c_{1}, c_{2}\right) \in W_{x_{i}}^{2}$, then $c_{1}, c_{2} \in W_{x_{i}}$, thus, $c_{1}, c_{2} \in Z$, this implies that $\left(c_{1}, c_{2}\right) \in N(\epsilon, Z) \times N(\epsilon, Z)$, which is a contradiction. Therefore, $C \cap W=\emptyset$.

Similarly, we obtain that $C^{*} \cap W=\emptyset$. Moreover, since $W=W^{*}$, it follows that $\left(C \cup C^{*}\right) \cap\left(W \cup W^{*}\right)=\emptyset$.

By Lemma 4.4, we have that $f_{2}(C)$ and $f_{2}(W)$ are two subcontinua of $F_{2}(X)$ such that $A \in \operatorname{int}_{F_{2}(X)}\left(f_{2}(C)\right) \subset F_{2}(X) \backslash F_{1}(X), F_{1}(X) \subset$ int $_{F_{2}(X)}\left(f_{2}(W)\right)$ and $f_{2}(C) \cap f_{2}(W)=\emptyset$. Hence, applying Remark 2.1, we conclude that $q_{X}^{2}\left(f_{2}(C)\right)$ and $q_{X}^{2}\left(f_{2}(W)\right)$ are two subcontinua of $S F_{2}(X)$ such that $\mathcal{A} \in \operatorname{int}_{S F_{2}(X)}\left(q_{X}^{2}\left(f_{2}(C)\right)\right), \mathcal{B} \in \operatorname{int}_{S F_{2}(X)}\left(q_{X}^{2}\left(f_{2}(W)\right)\right)$ and $q_{X}^{2}\left(f_{2}(C)\right) \cap$ $q_{X}^{2}\left(f_{2}(W)\right)=\emptyset$.

Finally, by Cases (1) and (2), we conclude that $S F_{2}(X)$ is mutually aposyndetic. 
TheOrem 4.7. If $X$ is a decomposable continuum, then $S F_{2}(X)$ is not strictly nonmutually aposyndetic.

Proof. Let $X$ be a decomposable continuum. Let $A_{1}$ and $A_{2}$ be proper subcontinua of $X$ such that $X=A_{1} \cup A_{2}$. Hence, there exist two points $x_{1} \in X \backslash A_{2}$ and $x_{2} \in X \backslash A_{1}$. We note that $x_{1} \in \operatorname{int}_{X}\left(A_{1}\right)$ and $x_{2} \in \operatorname{int}_{X}\left(A_{2}\right)$. Fix a number $\epsilon>0$ such that $\epsilon<\frac{1}{2} \min \left\{d\left(x_{1}, A_{2}\right), d\left(x_{2}, A_{1}\right)\right\}$. Consequently, by Lemma 3.9 , the set

$$
C=\left(A_{1} \times A_{2}\right) \backslash\left(N\left(\epsilon, A_{2}\right) \times N\left(\epsilon, A_{1}\right)\right)
$$

is a subcontinuum of $X^{2}$ such that $\left(x_{1}, x_{2}\right) \in$ int $_{X^{2}}(C)$ and $C \cap \triangle_{X^{2}}=\emptyset$.

We take $y \in N\left(A_{1}, \epsilon\right) \cap\left(X \backslash A_{1}\right)$. Since $N\left(A_{1}, \epsilon\right) \cap\left(X \backslash A_{1}\right)$ is an open subset of $X$, there exists an open subset $U$ of $X$ such that $y \in U \subset C l_{X}(U) \subset$ $N\left(A_{1}, \epsilon\right) \cap\left(X \backslash A_{1}\right)$. We define

$$
K=\left(C l_{X}(U) \times A_{2}\right) \cup\left(A_{2} \times C l_{X}(U)\right) .
$$

It follows that $K$ is a subcontinuum of $X^{2}$ such that $i n t_{X^{2}}(K) \neq \emptyset$.

Note that $\left(C \cup C^{*}\right) \cap\left(K \cup K^{*}\right)=\emptyset$. By Lemma 4.4, we have that $f_{2}(C)$ and $f_{2}(K)$ are subcontinua of $F_{2}(X)$ such that $\operatorname{int}_{F_{2}(X)}\left(f_{2}(C)\right) \neq \emptyset$, int $_{F_{2}(X)}\left(f_{2}(K)\right) \neq \emptyset$ and $f_{2}(C) \cap f_{2}(K)=\emptyset$.

Since $C \cap \triangle_{X^{2}}=\emptyset$, it follows that $f_{2}(C) \subset F_{2}(X) \backslash F_{1}(X)$. Applying Remark 2.1 we obtain that $q_{X}^{2}\left(f_{2}(C)\right)$ is a subcontinuum of $S F_{2}(X)$ such that $\operatorname{int}_{S F_{2}(X)}\left(q_{X}^{2}\left(f_{2}(C)\right)\right) \neq \emptyset$.

On the other hand, let $z \in U \backslash\{y\}$. Since $(y, z) \in U \times U \subset K,\{y, z\} \in$ $f_{2}(U \times U) \subset f_{2}(K)$. By [11, Lemma 9], we have that $f_{2}(U \times U)$ is an open subset of $F_{2}(X)$. Let $\mathcal{U}$ be an open subset of $F_{2}(X)$ such that $\{y, z\} \in \mathcal{U} \subset$ $f_{2}(U \times U) \cap\left(F_{2}(X) \backslash F_{1}(X)\right)$. Using Remark 2.1 we obtain that $q_{X}^{2}\left(f_{2}(\mathcal{U})\right)$ is an open subset of $S F_{2}(X)$ with $q_{X}^{2}(\{y, z\}) \in q_{X}^{2}\left(f_{2}(\mathcal{U}) \subset q_{X}^{2}\left(f_{2}(K)\right)\right.$. Thus, $q_{X}^{2}\left(f_{2}(K)\right)$ is a subcontinuum of $S F_{2}(X)$ such that $i n t_{S F_{2}(X)}\left(q_{X}^{2}\left(f_{2}(K)\right)\right) \neq \emptyset$.

Finally, since $f_{2}(C) \cap f_{2}(K)=\emptyset$ and $f_{2}(C) \subset F_{2}(X) \backslash F_{1}(X)$, by Remark 2.1 , we conclude that $q_{X}^{2}\left(f_{2}(C)\right) \cap q_{X}^{2}\left(f_{2}(K)\right)=\emptyset$. This prove that $S F_{2}(X)$ is not strictly nonmutually aposyndetic.

Theorem 4.8. Let $X$ a continuum with zero span. Then $X$ is indecomposable if and only if $S F_{2}(X)$ is strictly nonmutually aposyndetic.

Proof. Suppose that $X$ is indecomposable. Let $\mathfrak{A}$ be a subcontinuum of $S F_{2}(X)$ such that $i n t_{S F_{2}(X)}(\mathfrak{A}) \neq \emptyset$. We put $\mathcal{B}=\left(q_{X}^{2}\right)^{-1}(\mathfrak{A})$. Since $q_{X}^{2}$ is a monotone map, by $[21,2.2$, p.138], we obtain that $\mathcal{B}$ is a subcontinuum of $F_{2}(X)$, furthermore, we have that $\operatorname{int}_{F_{2}(X)}(\mathcal{B}) \neq \emptyset$. We take a component $W$ of $\left(f_{2}\right)^{-1}(\mathcal{B})$. Applying [11, Lemma 14] it follows that $i n t_{X^{2}}(W) \neq \emptyset$. Since $\pi_{1}$ and $\pi_{2}$ are open maps, we have that $\pi_{1}(W)$ and $\pi_{2}(W)$ are subcontinua of $X$ such that $\operatorname{int}_{X}\left(\pi_{1}(W)\right) \neq \emptyset$ and $\operatorname{int}_{X}\left(\pi_{2}(W)\right) \neq \emptyset$. Since $X$ is indecomposable, by [14, Corollary 1.7.21], it follows that $\pi_{1}(W)=X$ and $\pi_{2}(W)=X$. Since $X$ is a continuum with zero span, we conclude that 
$W \cap \triangle_{X^{2}} \neq \emptyset$. This implies that $\mathcal{B} \cap F_{1}(X) \neq \emptyset$. Consequently, $F_{X}^{2} \in \mathfrak{A}$. This prove that, $S F_{2}(X)$ is strictly nonmutually aposyndetic.

Applying Theorem 4.7 we obtain that if $S F_{2}(X)$ is strictly nonmutually aposyndetic, then $X$ is indecomposable.

It is known, for instance [10, p. 210], that if $X$ is a chainable continuum then $X$ is a continuum with zero span. Hence, by Theorem 4.8, we have the following corollary.

Corollary 4.9. Let $X$ a chainable continuum. Then $X$ is indecomposable if and only if $S_{2}(X)$ is strictly nonmutually aposyndetic.

\section{REFERENCES}

[1] F. Barrragán, On the $n$-fold symmetric product suspensions of a continuum, Topology Appl. 157 (2010), 597-604.

[2] F. Barrragán, Induced maps on $n$-fold symmetric product suspensions, Topology Appl. 158 (2011), 1192-1205.

[3] D. E. Bennett, Aposyndetic properties of unicoherent continua, Pacific J. Math. 37 (1971), 585-589.

[4] K. Borsuk and S. Ulam, On symmetric products of topological space, Bull. Amer. Math. Soc. 37 (1931), 875-882.

[5] J. F. Davis, The equivalence of zero span and zero semispan, Proc. Amer. Math. Soc. 90 (1984), 133-138.

[6] J. T. Goodykoontz, Jr., Aposyndetic properties of hyperspaces, Pacific J. Math. 27 (1973), 91-98.

[7] H. Hosokawa, Mutual aposyndesis in n-fold hyperspaces, Houston J. Math. 35 (2009), $131-137$.

[8] F. B. Jones, Aposyndetic continua and certain boundary problems, Amer. J. Math. 63 (1941), 545-553.

[9] H. Katsuura, Characterization of arcs by products diagonals, manuscript.

[10] A. Lelek, Disjoint mappings and the span of spaces, Fund. Math. 55 (1964), 199-214.

[11] S. Macías, Aposyndetic properties of symmetric products of continua, Topology Proc. 22 (1997), 281-296.

[12] S. Macías, On the hyperspaces $C_{n}(X)$ of a continuum $X$, Topology Appl. 109 (2001), $237-256$.

[13] S. Macías, On the n-fold hyperspace suspension of continua, Topology Appl. 138 (2004), 125-138.

[14] S. Macías, Topics on Continua, Pure and Applics Mathematics Series, Vol. 275, Chapman \& Hall/CRC, Taylor \& Francis Group, 2005.

[15] J. C. Macías, On n-fold pseudo-hyperspace suspensions of continua, Glas. Mat. Ser. III 43 (2008), 439-449.

[16] J. M. Martínez-Montejano, Mutual aposyndesis of symmetric products, Topology Proc. 24 (1999), 203-213.

[17] S. B. Nadler, Jr., Continua which are an one-to-one continuous image of $[0, \infty)$, Fund. Math. 75 (1972), 123-133.

[18] S. B. Nadler, Jr., Hyperspaces of Sets, Monographs and Textbooks in Pure and Applied Math., Vol. 49, Marcel Dekker, New York-Basel, 1978.

[19] S. B. Nadler, Jr., Continuum theory. An introduction, Monographs and Textbooks in Pure and Applied Math., Vol. 158, Marcel Dekker, New York, 1992. 
[20] L. E. Rogers, Mutually aposyndetic products of chainable continua, Pacific J. Math. 37 (1971), 805-812.

[21] G. T. Whyburn, Analytic Topology, Amer. Math. Soc. Colloq. Publ., vol. 28, Amer. Math. Soc., Providence, R. I., 1942.

F. Barragán

Instituto de Física y Matemáticas

Universidad Tecnológica de la Mixteca

K. M. 2.5 Carretera Huajuapan-Acatlima, Huajuapan de León, Oaxaca, C.P. 69000

México

E-mail: frabame@hotmail.com

Received: 2.6.2013.

Revised: 22.8.2013. \& 5.9.2013. 